\title{
Lean Logistics - Innovative Bewertungsmethodik zur Auswahl schlanker Materialbereitstellungsstrategien
}

\author{
Prof. Dr.-Ing. Nina Vojdani, Dipl.-Wirt.-Ing. Felix Lootz, \\ Dipl.-Wirt.-Ing. Dennis Kirwitzke \\ Universität Rostock \\ Lehrstuhl für Produktionsorganisation und Logistik
}

\begin{abstract}
Die starke Individualisierung der Kundenwünsche hat eine stetig wachsende Variantenvielfalt zur Folge. Unternehmen, insbesondere aus dem Automotive- und High-Tech-Sektor, sind bestrebt, ein dauerhaftes Vorhalten aller Variantenteile im Montageprozess aufgrund hoher Kapitalbindung, zunehmenden Flächenbedarfs sowie steigenden Handlingaufwands zu vermeiden. Durch die konsequent wertschöpfungsorientierte Planung der Montageprozesse ist es möglich, innerhalb eines Montagetakts größere Teileumfänge zu verbauen. Das Angebot an Bereitstellflächen erhöht sich jedoch nicht, so dass Kosteneinsparungen aus Montageoptimierungen zum Teil nicht ausgeschöpft werden können. Diese Einsparungen können erst zusammen mit einem angepassten und standardisierten Materialbereitstellungsprozess voll wirksam werden. Die Materialbereitstellung muss darüber hinaus auch für individuelle Teilespektren eine adäquate Teileversorgung sichern sowie innerhalb ihrer Systemgrenzen einen optimalen Prozessablauf ermöglichen. Für die Auswahl geeigneter Bereitstellungsstrategien fehlt eine auf den Bereitstellungsprozess abgestimmte Bewertungsmethode, die eine nach Lean Management Grundsätzen vergleichende Prozessbetrachtung ermöglicht und zudem bauteilspezifische Kriterien wie Variantenvielfalt, Reichweite oder Größe berücksichtigt. Ziel des Beitrags ist es daher, eine neuartige Bewertungsmethode vorzustellen.
\end{abstract}

Keywords: lean logistics, material handling, material provisioning processes

\section{$1 \quad$ Motivation und Zielsetzung}

Vor allem in gesättigten Märkten, wie dem Automotive- und High-Tech-Sektor, ist der Kunde der bestimmende Faktor. Aufgrund verschiedener Kombinationsmöglichkeiten der zahlreichen Ausstattungsvarianten stellt beispielsweise im Automobilbau mittlerweile nahezu jedes hergestellte Fahrzeug ein Unikat dar. Diese Entwicklung hat die Komplexität und Variantenvielfalt der Bauteile enorm erhöht. Dadurch steigen zum einen die eigentlichen Montage- und Herstellkosten, zum anderen wirkt sich das ständige Vorhalten aller Variantenteile, durch hohe Kapitalbindung, hohes Verschrottungsrisiko, zunehmenden Flächenbedarf sowie steigenden Handlingaufwand kostenerhöhend aus. Betroffene Unternehmen verfolgen deshalb das Ziel, die Abläufe sowohl in den Fertigungs- als auch in den fertigungsnahen Bereichen bei steigenden Flexibilitäts- und Qualitätsanforderungen effizienter und produktiver zu gestalten. 
Ein Ansatz dazu findet sich in Produktionssystemen, die nach den Grundsätzen des Lean-Managements arbeiten. Sie zeichnen sich durch eine effiziente Prozessgestaltung sowie eine stetige Ausrichtung der Produktion am Kundenbedarf aus. Durch die konsequent wertschöpfungsorientierte Planung und der damit einhergehenden Erhöhung des Wertschöpfungsanteils in den Montageprozessen ist es möglich, innerhalb eines Montagestakts größere Teileumfänge zu verbauen. Das Angebot an Bereitstellflächen erhöht sich jedoch nicht, so dass Kosteneinsparungen aus Montageoptimierungen zum Teil nicht ausgeschöpft werden können. Positive Effekte aus Verbesserungen im Montageprozess werden erst zusammen mit einem angepassten Materialbereitstellprozess voll wirksam. Es ist deshalb notwendig, die Materialbereitstellung abhängig vom Montageprozess entsprechend den Prinzipien des Lean-Managements zu untersuchen und neue Bereitstellungsstrategien zu entwickeln. Daraus sollen standardisierte Bereitstellungsstrategien entstehen, die bezüglich des jeweils bereitzustellenden Teilespektrums eine adäquate Teileversorgung sichern sowie innerhalb festgelegter Systemgrenzen den optimalen Prozessablauf der Materialbereitstellung darstellen.

Für die Auswahl geeigneter Bereitstellungsstrategien ist eine auf den Prozess abgestimmte Bewertungsmethode notwendig, die eine nach Lean ManagementPrinzipien vergleichende Prozessbetrachtung ermöglicht und zudem bauteilspezifische Kriterien wie Variantenvielfalt, Reichweite oder Größe berücksichtigt. Nach dem Stand der Forschung sind hierfür kaum Ansätze identifizierbar. Bullinger [Bul94] beispielsweise fokussiert auf Leitlinien zur Arbeitsgestaltung der Materialbereitstellung und schwerpunktmäßig auf deren technischen Bewertung. Weiterhin macht Bullinger Ausführungen zur bauteilabhängigen Einteilung eines Teilespektrums, integriert diese jedoch nicht in eine Bewertungsmethode. Vor dem Hintergrund einer zeitlichen und monetären Bewertung betrachtet Grünz [Grü04] lediglich das Fehlerrisiko bei Materialbereitstellungsprozessen. Weitere Autoren, bspw. Lotter [LW06], bewerten die Prozessgestaltung mittels einer Nutzwertanalyse, deren Methodik für die gegebene Problemstellung kaum geeignet ist, da in dem gegebenen Kontext eine Gewichtung von Kriterien nicht notwendig ist.

Ziel des Beitrags ist daher die Vorstellung einer innovativen Bewertungsmethodik zur bedarfsgerechten Auswahl schlanker Materialbereitstellungsstrategien. Im Folgenden erfolgt dazu eine Beschreibung der Grundsätze einer schlanken Materialbereitstellung sowie eine prozessorientierte Beschreibung ausgewählter Materialbereitstellungsstrategien. Darauf aufbauend wird eine ganzheitliche Bewertungsmethode systematisch hergeleitet und präsentiert. Abschließend werden die Erfahrungen eines Pilotprojekts vorgestellt und die Ergebnisse dieses Beitrags zusammengefasst. Der Beitrag basiert auf Erfahrungen und gewonnenen Erkenntnissen aus einem Projekt in der VFlex Motorenmontage der BMW Group. 


\section{Grundsätze einer schlanken Materialbereitstellung}

Die Materialbereitstellung ist eng mit den angrenzenden Prozessen der Materialversorgung und des Materialabgriffs verknüpft. Diese müssen bei der Erarbeitung der Bereitstellungsstrategien herangezogen werden. Es gilt, einen von beiden Prozessen abhängigen, optimalen Prozess zu finden, der sich nicht nur nach einem lokalen Optimum orientiert, sondern ein möglichst gesamtoptimales Ergebnis gewährleistet. In diesem Beitrag ist der Blick auf die Gestaltung und Bewertung von Materialbereitstellungsstrategien gerichtet, zudem findet die Anbindung angrenzender Prozesse Beachtung. Somit beeinflusst der Materialabgriff die Beurteilung der Materialbereitstellung. Gleichzeitig werden Gestaltungsprinzipien der Materialversorgung berücksichtigt. Die Materialversorgung außerhalb des Montagesystems wird beim betrachteten Automobilhersteller nach 3 Strategien durchgeführt: Direktanlieferung, Anlieferung aus Logistikzentrum (automatisches Kleinteilelager mit Kleinladungsträger [KLT]) und Anlieferung aus Versorgungszentrum (Hochregallager für Großladungsträger [GLT]). Nach der LeanPhilosophie ist die Direktanlieferung taktgenau, just-in-time und in Standard-KLT und -GLT nach dem Pullprinzip durchzuführen. Diese Strategie ist in der Regel nur für bestimmte Teilegruppen anwendbar und stellt daher eher eine Ideallösung dar. Die später aufgezeigten Materialbereitstellungsstrategien werden nach GLT und KLT differenziert, was zu einer Reduzierung der Einsatzmöglichkeiten der Strategien auf bestimmte Teilespektren führt.

Zusätzlich zu den Rahmenbedingungen spielen die Prinzipien einer schlanken Materialbereitstellung bei der Erarbeitung und Auswahl der Materialbereitstellungsstrategien eine wichtige Rolle. In der Abbildung 1 sind die Gestaltungsprämissen zusammengefasst: Dazu zählen die Prinzipien der LeanProduction, die Ziele schlanker Materialbereitstellung und die Gestaltungsanforderungen der Variantenfertigung von Motoren. 


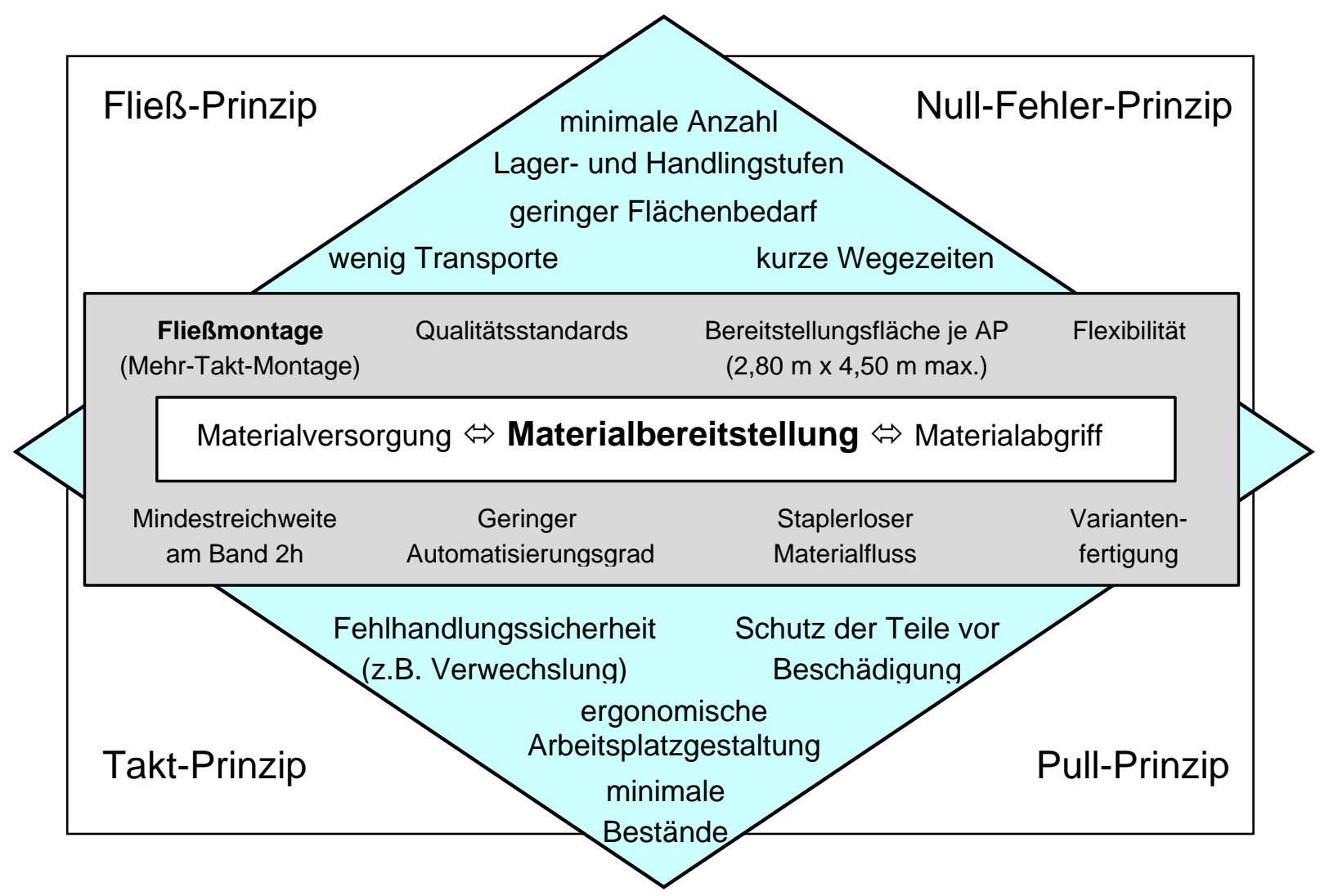

Abbildung 1: Grundsätze einer schlanken Materialbereitstellung

Bei der Erarbeitung der Materialbereitstellstrategien wird ein großes Augenmerk auf die Gestaltung eines optimalen Materialabgriffs gelegt; daher gelten für beide Prozesse die gleichen Gestaltungsprämissen. Dabei ist besonders auf eine ergonomische Gestaltung für den Abgriff des Materials zu achten. Das bedeutet, dass bei der Handhabung keine unnatürlichen Körperhaltungen (z.B. Bücken, Strecken, Drehen) eingenommen werden sollten. Zusätzlich beinhaltet ein optimaler Materialabgriff kurze Griffweiten und einen fehlhandlungssicheren Teilezugriff für den Montagemitarbeiter (MMA). Für kurze Wegezeiten des Mitarbeiters wird möglichst ein Ein-Punkt-Abgriff angestrebt, d.h. der Teileabgriff je Arbeitsplatz erfolgt an einem Bereitstellungsort. Für die Bereitstellung innerhalb des Montagesystems wird von einer getakteten, standardisierten Versorgung durch einen Routenzug ausgegangen.

\section{$3 \quad$ Bereitstellungsstrategien nach der Lean-Philosophie}

Die VFlex Motorenmontage bildet die Grundlage zur Erarbeitung der Bereitstellungsstrategien. Es handelt sich um eine getakte Fließmontage, in der ein MMA in einer Arbeitsplatzgruppe 6 Takte bearbeitet. Die Bereitstellung erfolgt über ein Engineset, welches vor dem ersten Takt an die Werkstückaufnahme angekoppelt wird und während der Montage mitläuft. Es handelt sich dabei um eine Plattform, auf der Bauteile motorbezogen bereitgestellt werden können. Des Weiteren ist die Bereitstellung direkt am Montageband möglich. 
Die Gestaltungsgrundsätze bilden die Basis für die Erarbeitung der Bereitstellungsprozesse. Dabei wurde der Verlauf der Materialbereitstellung ausgehend vom Materialabgriff von innen nach außen innerhalb der Betrachtungsgrenzen entwickelt. Die aus den Bereitstellungsprozessen abgeleiteten Bereitstellstrategien sollen alternative und schlanke Ansätze der Materialbereitstellung darstellen. Im Zuge der Forschung wurden sechs innovative Strategien erarbeitet, dazu gehören:

- Kommissionieren in PickBox über Supermarkt am Montageband

- Kommissionieren in SetBox über bandnahen Supermarkt

- KLT Bereitstellung über dem Arbeitsplatz.

Im Folgenden wird exemplarisch die Strategie PickBox in Kleinteile-Supermarkt (SuMa) am Montageband näher beschrieben.

Die Strategie beinhaltet die Kommissionierung von Kleinteilen in eine PickBox direkt am Montageband. Die PickBox ist ein in Fächer unterteilter Behälter für Kleinteile, versehen mit einem Tragegriff. Im Idealfall lässt sich die Facheinteilung durch verschiedene Einsätze variabel gestalten. Es ist vorgesehen, dass der MMA für die Tätigkeiten in einer Montageplatzgruppe die Klein- und Normteile aus einer PickBox abgreift, die er auf dem Engineset abstellt. Die Teile befinden sich so für die MMA immer in einer Griffweite von 0,50 bis $0,80 \mathrm{~m}$. Auf dem Rückweg zum ersten Montageplatz füllt der Mitarbeiter die Box in einem Supermarkt direkt am Montageband auf. Für den Transport der PickBoxen ist am SuMa eine Rollenbahn angebracht, die zu einem Pufferplatz auf Höhe des ersten Montageplatz führt. Dort stehen die Boxen für den nächsten Motor bereit. Der Inhalt der Boxen unterscheidet sich je nach Motortyp. Der Vorteil dieser Strategie liegt in der Ausnutzung des vorhandenen Rückweges des MMA für das Auffüllen der Boxen. Als zusätzlicher Aufwand ist lediglich die Zeit für das Picken der Teile zu berücksichtigen. Die Griffweite ist gegenüber der konventionellen Bereitstellung (KLT im Durchlaufregal) um die Hälfte auf ca. 0,80 m verkürzt, außerdem entfallen Körperdrehungen (vgl. Abschnitt 5). Dazu kommen Bestandsreduzierungen, da jedes Teil innerhalb der Montageplatzgruppe nur einmal bereitgestellt werden muss. Bei Änderungen der Arbeitsvorgangsfolge innerhalb der Montageplatzgruppe sind keine Umstellungen in der Bereitstellung notwendig. Als geringer Nachteil ist die Erhöhung der logistischen Tätigkeiten des MMA zu sehen. Diese Strategie lässt sich auf alle Bauteile kleiner und mittlerer Größe anwenden, die aus Qualitätssicht ein Umfüllen in die PickBox gestatten und den Fachabmessungen entsprechen, Abbildung 2. 


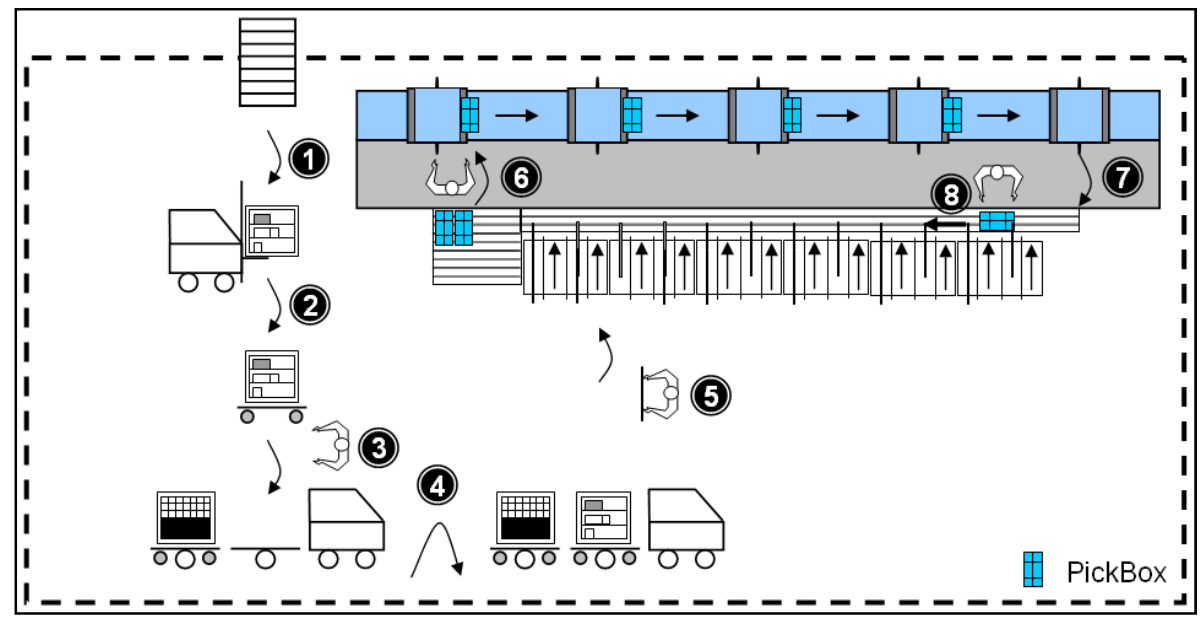

Abbildung 2: Kommissionieren über PickBox in Supermarkt am Montageband

\section{Bewertungsmethode zur bauteilspezifischen Auswahl von Materialbereitstellungsstrategien}

Neben der Identifikation und Entwicklung alternativer Materialbereitstellungsstrategien stehen die Unternehmen vor der Herausforderung, daraus die optimale Materialbereitstellung auszuwählen. Vor diesem Hintergrund wurde durch den Lehrstuhl eine auf den Bereitstellungsprozess abgestimmte Bewertungsmethode entwickelt, die eine nach Lean Management Grundsätzen vergleichende Prozessbetrachtung ermöglicht und zudem bauteilspezifische Kriterien berücksichtigt. Sowohl der Bereitstellungsprozess als auch die bereitzustellenden Bauteile beeinflussen die Beurteilung der Materialbereitstellungsstrategien, daher baut die Bewertungsmethode auf zwei Modulen auf, Abbildung 3:

Das „Modul - Prozesssicht" befasst sich ausschließlich mit der Bewertung der Prozessabläufe von Materialbereitstellungsstrategien. Das "Modul - Produktsicht“ bewertet die Eignung einer Strategie bezüglich bauteilabhängiger Kriterien. Die Ergebnisse beider Module werden in einer Bewertungsmatrix zusammengeführt. 


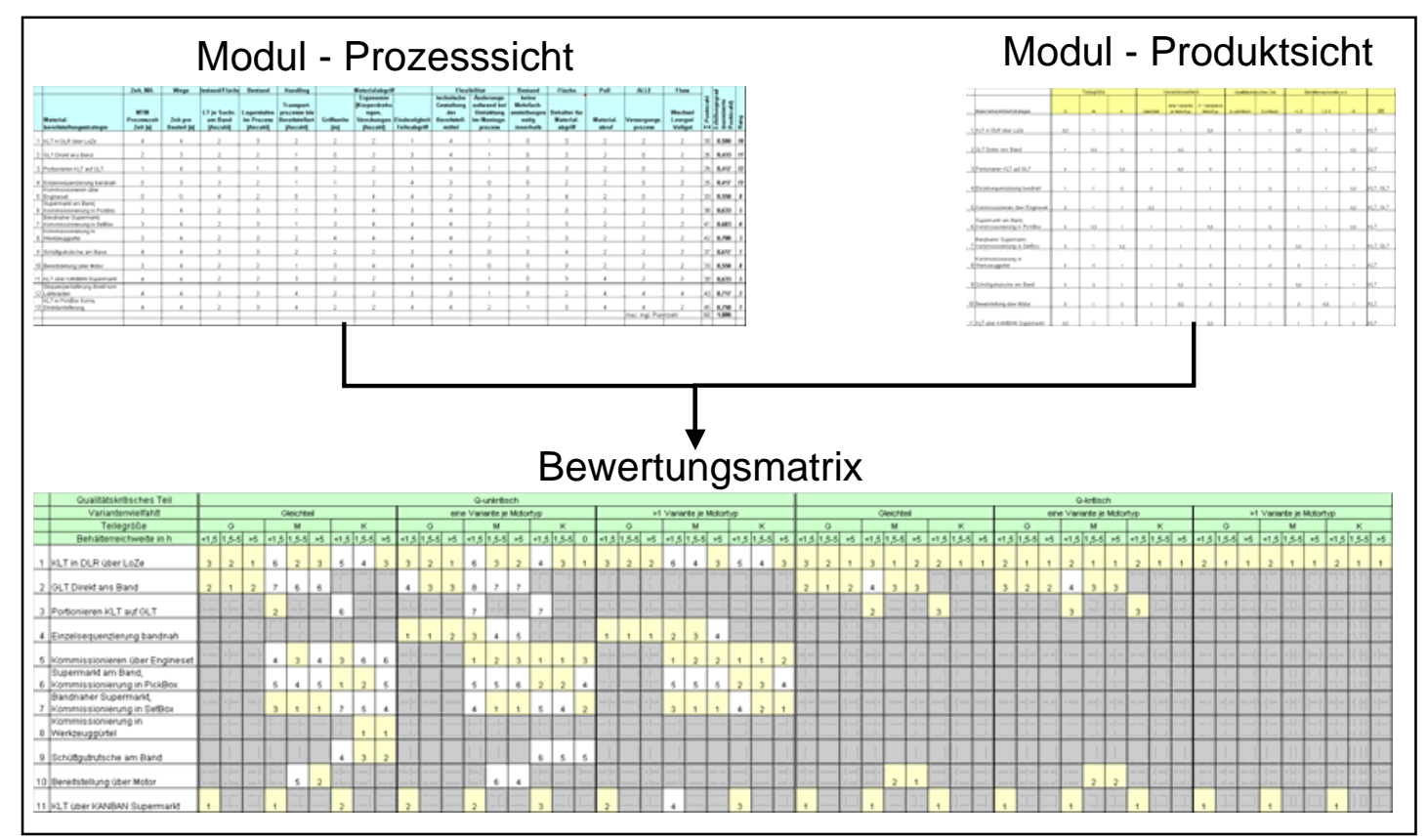

Abbildung 3: Bewertungsmethode zur Auswahl von Bereitstellungsstrategien

Zur Bewertung der Prozessgestaltung im „Modul - Prozesssicht“ wurden Kriterien ausgewählt, die die Grundsätze einer schlanken Materialbereitstellung repräsentieren. Dazu zählen bspw. die MTM Prozesszeit je Ladungsträger, Lagerstufen im Prozess, Griffweite sowie Eindeutigkeit des Teileabgriffs. Die Ausprägungsstufen der Kriterien können als unterschiedliche Zahlenwerte (z.B. 1 m, 24 s) auftreten oder als Zustandsbeurteilung wörtlich charakterisiert sein (z.B. „flexibel“, „unflexibel“). Die Bewertungsmethodik hat die Aufgabe diese zusammenzufassen und eine vergleichbare Bewertung zu ermöglichen. Einfachheit und Praxisnähe sind zwei weitere Prämissen. Die einfache Punktbewertung nach VDI 2225 (Punkteskala von 4 bis 0 Punkten) ist dafür eine geeignete Methode, da sich mit ihr sowohl quantitative als auch qualitative Kriterien in ein gemeinsames Bewertungsschema bringen lassen.

Die verschiedenen Ausprägungen aller Kriterien werden den Bewertungspunkten zugeordnet. Im nächsten Schritt werden für die Prozessabläufe der Bereitstellungsstrategien die Ausprägungsstufen je Bewertungskriterium identifiziert. Daraus ergibt sich eine Einzelpunktzahl je Kriterium. Die Summe der Einzelpunktzahlen bildet eine Gesamtpunktzahl je bewerteten Prozess. Sie lässt bereits eine vergleichende Betrachtung zu. Um eine Vergleichbarkeit mit Ergebnissen späterer Bewertungen zu ermöglichen, wird die Gesamtpunktzahl auf Basis der maximal möglichen Punkte normiert.

Das „Bewertungsmodul - Produktsicht" berücksichtigt den Einfluss von Bauteileigenschaften auf die Auswahl von Materialbereitstellungsstrategien. Die Prämissen für die Auswahl der vier Bewertungskriterien leiten sich aus den LeanGrundsätzen ab. Die Variantenvielfalt eines Bauteils beeinflusst den Flächendedarf und leitet sich aus dem Fließ-Prinzip ab, Qualitätsrelevanz berücksichtigt das NullFehler-Prinzip, Bauteilgröße und Behälterreichweite haben Ihre Grundlage im (c) 2010 Logistics Journal : Proceedings - ISSN 2192-9084 
Grundsatz der getakteten Versorgung. Anhand dieser Kriterien werden BasisTeileklassen gebildet, aus denen sich die Teileklassen zusammensetzen. Das Kriterium Bauteilgröße wird bspw. in die Basis-Teileklassen große, mittlere und kleine Bauteile unterteilt und spiegelt die Aufnahmefähigkeit der Bereitstellungsmittel wider. Durch die Kombination von Basis-Teileklassen werden Teileklassen gebildet, z.B. "Gleichteil-Kleinteil-Reichweite>5h“. Die Bewertung der Eignung einer bestimmten Teileklasse wird mit Gewichtungsfaktoren erreicht. Diese reflektieren die Realisierbarkeit eines Prozesses und haben die Ausprägungen 0, 0.5 und 1, Abbildung 4.

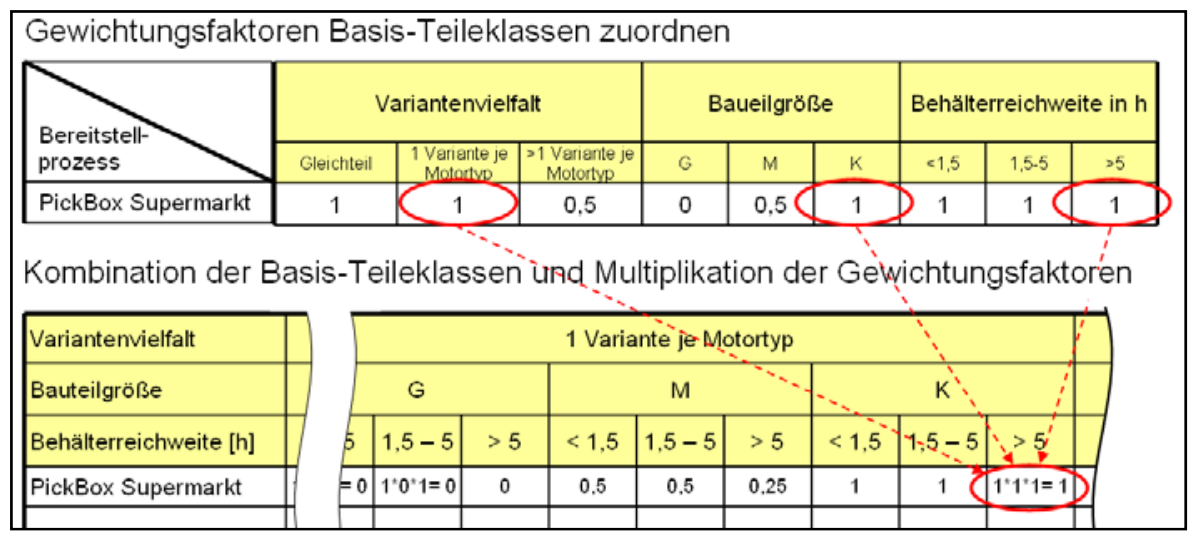

Abbildung 4: Zusammensetzung der Teileklassenmatrix im Modul - Produktsicht

Aus der Prozessbeschreibung sind im ersten Schritt für jeden zu bewertenden Prozess die Gewichtungsfaktoren je Basis-Teileklasse abzuleiten. Für die Bildung einer Auswahlmatrix werden zusammengefasste Teileklassen benötigt. Diese werden wie oben beschrieben durch die Kombination der Basis-Teileklassen geschaffen. Die gleichzeitige Multiplikation der entsprechenden Gewichtungsfaktoren der Basis-Teileklassen bildet Bewertungsfaktoren, die in einer Teileklassenmatrix den Bereitstellungsstrategien zugeordnet sind. Die Bewertungsfaktoren bilden den zweiten Bestandteil der Bewertungsmatrix. Durch die multiplikative Verknüpfung der Gewichtungsfaktoren ergeben alle nicht möglichen Kombinationen von Teileklassen und Prozessen den Wert Null und gehen nicht in die weitere Bewertung ein. Anschließend erfolgt die Zusammenführung der Kennzahlen beider Module zur Bewertungsmatrix.

Den Aufbau der Bewertungsmatrix legt dabei die Teileklassenmatrix fest. Die darin erhaltenen Bewertungsfaktoren werden je Bereitstellstrategie mit der Bewertungspunktzahl des „Moduls - Prozesssicht“ multipliziert. Aus diesen Bewertungskennzahlen wird in einem letzten Schritt eine Rangfolge der Bereitstellungsstrategien je Teileklasse ermittelt und in einer Auswahlmatrix dargestellt. Nicht geeignete Prozesse (Bewertungsfaktor $=0$ ) sind in der Matrix in einem leeren, grau hinterlegten Feld dargestellt.

Um eine zu einer Teileklasse passende Strategie auszuwählen wird entsprechend der Bauteilcharakteristik die Teileklasse im oberen Bereich der Matrix lokalisiert. In 
der entsprechenden Spalte wird die Zeile mit dem besten Rang und einem übereinstimmendem Behältertyp identifiziert. Anschließend wird in der linken Spalte die entsprechende Bereitstellstrategie abgelesen.

\section{$5 \quad$ Aufbau eines Pilotarbeitsbereichs}

Auf der Grundlage der erarbeiteten Bewertungsmethode zur der Materialbereitstellung wurde in einem Pilotarbeitsbereich die praktische Umsetzung vorgenommen. Das Ergebnis der Anwendung der Bewertungsmatrix war für das vorhandene Teilespektrum die Auswahl der „Kommissionierung in PickBox über SuMa am Montageband" als optimale Bereitstellungsstrategie. Auf diese Bereitstellungsform wurde der Großteil der Bauteile verlagert. Der PickBox-SuMa ersetzt vollständig vorhandene Durchlaufregale. Die Entwicklungsstufen des SuMa werden im Folgenden beschrieben.

Bereits im Zuge der Erarbeitung der Bereitstellungsstrategien wurde ein erster Prototyp eines Kleinteile-SuMa für die Füllung von PickBoxen am Montageband konstruiert und aufgebaut. Dieser Prototyp bildete die Basis für die Entwicklung des SuMa und für die praktische Umsetzung der Bereitstellungsstrategie. Im nächsten Schritt wurde der SuMa im Rahmen einer Workshoparbeit abseits vom Montageband als Simulation aufgebaut und der Ablauf im Team sowie mit den Mitarbeitern getestet und optimiert. Zu diesem Zeitpunkt wurde auch die endgültige Füllung der PickBoxen festgelegt. Vor dem SuMa ist eine Rollenbahn angebracht, auf der der Mitarbeiter auf dem Rückweg, beim Befüllen der Box, diese abstellen und schieben kann. So wird eine unergonomische Belastung durch das Tragen der PickBox verhindert. Am Ende der Rollenbahn befindet sich eine Pufferfläche für die PickBoxen, die auch aus Rollenbahnen aufgebaut ist und auf der nicht benötigte Boxen abgestellt werden. Im dritten Schritt wurde die Materialbereitstellung an bestimmten Arbeitsplätzen entsprechend der gewonnenen Erkenntnisse umgebaut. In dieser Phase der Umsetzung wurden motorspezifische Markierungen (Motorbezeichnung, MotorFarbcode) an den Außenseiten der PickBox sowie Bauteilbeschriftungen (Bauteilbezeichnung, Sachnummer sowie Motor-Farbcode) in den Boxenfächern angebracht.

Im Vergleich zur vorherigen Situation wurde für knapp 60 Bauteile die Bereitstellung auf die PickBox umgestellt. Abgesehen von der Schaffung eines harmonischen, fließenden Gesamtprozesses in Montage und Logistik ergeben sich daraus einige Kennzahlen, die den Erfolg der Umbaumaßnahmen und somit den praktischen Nutzen der Bewertungsmatrix zeigen. Die Zahlenwerte beziehen sich jeweils auf eine Schicht. Durch Optimierung der Bereitstellung wurde für die MMA beim Materialabgriff eine Einsparung von insgesamt über $3 \mathrm{~km}$ Weg und mehr als 2000 Körperdrehungen erreicht. Die Einrichtungen des PickBox-SuMa bedingt zusätzlich eine abtaktungsneutrale Bereitstellung, die die Flexibilität für den Montageprozess erhöht. Durch das Verlagern von weiteren Bauteilen auf das Engineset und das 
Eliminieren von Doppellagerungen im Bereich des PickBox-SuMa wurde zudem eine Bestandsreduzierung am Montageband erreicht.

\section{$6 \quad$ Fazit}

Die Grundlagen einer effizienten und wertschöpfungsorientierten Materialbereitstellung liegen zum einem in einem stabilen und verschwendungsfreien Montageprozess und berücksichtigen zum anderen die Prozesse der Materialversorgung und des Materialabgriffs. Nur eine ganzheitliche Betrachtung und Optimierung kann eine effiziente Materialbereitstellung gewährleisten. Weiterhin können Unternehmen durch fehlerfreie, reibungslose und verschwendungsfreie Materialbereitstellungsprozesse der zunehmenden Variantenvielfalt begegnen.

Die entwickelte Bewertungsmethode wird dem Anspruch gerecht, die Bereitstellungsprozesse entsprechend den Grundsätzen einer schlanken Materialbereitstellung zu beurteilen. Die Bewertung folgt einer einfachen Methodik und kann mit geringem Aufwand auf andere Montagesysteme adaptiert werden.

Dr. Dieter Geus, Initiator des Projektes auf Seiten der BMW Group resümiert die praktischen Relevanz folgendermaßen: „In der Logistikplanung der BMW Motorenmontage ist die Bewertungsmethode mittlerweile Grundlage der schlanken Materialbereitstellplanung geworden, mit positiven Effekten sowohl auf den Planungsprozess als auch auf die Planungsergebnisse."

Nicht nur inhaltlich findet der Lean-Ansatz in der Bewertungsmethode Anwendung, auch der Aufbau der Methodik ist schlank, flexibel und praxisbezogen gestaltet.

\section{Literatur}

[Bul94] Bullinger, H.-J.; Lung, M.: Planung der Materialbereitstellung in der Montage. Stuttgart: Teubner, 1994.

[Grü04] Grünz, L.: Ein Modell zur Bewertung und Optimierung der Materialbereitstellung. Aachen: Shaker, 2004.

[LW06] Lotter, B.; Wiendahl, H.-P.: Montage in der industriellen Produktion. Ein Handbuch für die Praxis. Berlin: Springer, 2006. 